\title{
Fratura bilateral do colo do fêmur secundária a crise convulsiva: Tratamento com artroplastia total do quadril pelo acesso anterior direto*
}

\section{Bilateral Femoral Neck Fracture Secondary to Seizure: Treatment with Total Hip Arthroplasty by the Direct Anterior approach}

\author{
Osamu de Sandes Kimura ${ }^{1}$ Rui Felipe Pache de Moraes ${ }^{1}$ Marco Bernardo Cury Fernandes ${ }^{10}$ \\ Emílio Henrique Carvalho Freitas ${ }^{1}$ Alexandre Seabra ${ }^{1}$ George Kalif Lima ${ }^{1}$
}

${ }^{1}$ Instituto Nacional de Traumatologia e Ortopedia (Into), Rio de Janeiro, RJ, Brasil

Rev Bras Ortop 2020;55(2):254-257.
Endereço para correspondência Osamu de Sandes Kimura, Instituto Nacional de Traumatologia e Ortopedia (Into), Rio de Janeiro, RJ, Brasil (e-mail: osamukimura@gmail.com).

\section{Resumo \\ Palavras-chave \\ - fraturas do colo femoral \\ - artroplastia de quadril \\ - convulsões \\ - epilepsia}

\section{Abstract}

A fratura bilateral do colo do fêmur secundária à crise convulsiva é um evento raro. A ocorrência dessas lesões está relacionada a contrações musculares tônico-clônicas vigorosas e ao uso de medicações anticonvulsivantes. As fraturas do colo do fêmur no adulto jovem tratadas com artroplastia total do quadril são exceção, e a escolha do acesso cirúrgico deve levar em consideração diversos fatores; o acesso anterior direto é uma possibilidade para artroplastia total do quadril. Os autores apresentam o caso de um homem de 36 anos com fratura bilateral do colo do fêmur secundária a crise convulsiva e em uso regular de fenitoína. Devido ao risco de falha da fixação e ao tempo de evolução prolongado, optou-se pela artroplastia total do quadril bilateral. A escolha da via de acesso deve levar em consideração a anatomia do paciente, a disponibilidade de materiais e a experiência do cirurgião. Dessa forma, a maior facilidade de preparo e posicionamento do paciente, o menor tempo de internação, a reabilitação pósoperatória precoce e o domínio da técnica pelo cirurgião são possíveis justificativas para a adoção do acesso anterior direto.

Bilateral fracture of the femoral neck secondary to seizure is a rare event. The occurrence of these lesions is related to vigorous tonic-clonic muscular contractions and to the use of anticonvulsive medications. Femoral neck fractures in young adults treated with total hip arthroplasty are the exception and the choice of surgical access should take into account several factors; the direct anterior approach is a possibility for total hip arthroplasty.

\footnotetext{
* Trabalho desenvolvido no Centro de Cirurgia do Quadril, Instituto Nacional de Traumatologia e Ortopedia (Into), Rio de Janeiro, RJ, Brasil. Originalmente publicado pela Elsevier Editora Ltda.
}

recebido

19 de Setembro de 2017 aceito 14 de Dezembro de 2017
DOI https://doi.org/

10.1016/j.rbo.2017.12.024. ISSN 0102-3616.
Copyright $\odot 2020$ by Sociedade Brasileira License terms de Ortopedia e Traumatologia. Published by Thieme Revinter Publicações Ltda, Rio de Janeiro, Brazil 


\author{
Keywords \\ - femoral neck \\ fractures \\ - arthroplasty, \\ replacement, hip \\ - seizure \\ - epilepsy
}

The authors present the case of a 36-year-old male with bilateral fracture of the femoral neck secondary to seizure and in regular use of phenytoin. Due to the risk of fixation failure and prolonged evolution time, bilateral total hip arthroplasty was the procedure of choice. The choice of the approach should take into consideration the patient's anatomy, material availability, and surgeon's experience. Thus, the greater ease of preparation and positioning of the patient, the shorter hospital stay, the early postoperative rehabilitation, and the mastery of the technique by the surgeon are possible justifications for the adoption of the direct anterior approach.

\section{Introdução}

A fratura bilateral do colo do fêmur secundária a crise convulsiva é um evento raro, com poucos casos descritos na literatura. ${ }^{1-3} \mathrm{~A}$ ocorrência dessas lesões está relacionada a contrações musculares tônico-clônicas vigorosas que geram fraturas ou luxações nos segmentos proximais dos membros. ${ }^{1}$ Além disso, o uso de medicações anticonvulsivantes leva a alterações na matriz óssea que predispõem a ocorrência de fraturas. ${ }^{4,5}$

As fraturas do colo do fêmur no adulto jovem são preferencialmente tratadas com fixação; a artroplastia total de quadril é indicada em casos de exceção. ${ }^{6}$ A escolha do acesso cirúrgico deve levar em consideração diversos fatores, a via anterior direta é um possível acesso para artroplastia do quadril.

Nosso objetivo é relatar o caso de um paciente jovem com fratura bilateral do colo do fêmur pós-crise convulsiva, manejado com artroplastia total do quadril pela via anterior direta.

\section{Relato de Caso}

Paciente de 36 anos, masculino, branco, e trabalhava como motoboy. História prévia de traumatismo crânio encefálico havia 4 anos, submetido a procedimentos neurocirúrgicos. Referia episódios convulsivos desde então, em uso contínuo de fenitoína e acompanhamento neurológico regular, porém persistia com episódios convulsivos.

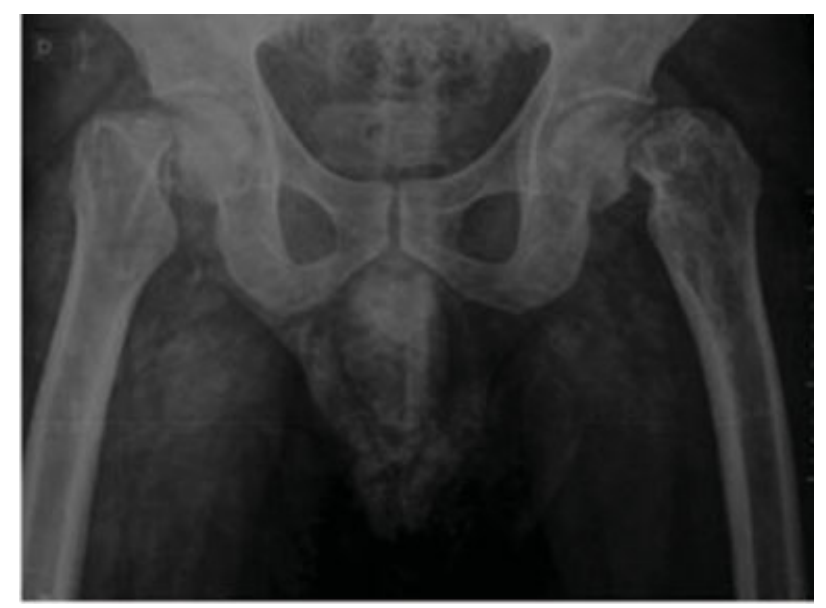

Fig. 1 Radiografia anteroposterior da bacia pré-operatória.
O paciente foi encaminhado ao serviço com 30 dias de evolução devido a fratura bilateral do colo do fêmur secundária a crise convulsiva. Inicialmente atendido em sua cidade de origem, o paciente apresentava dor e limitação funcional em ambos os membros. Negava história de trauma associado, porém a crise convulsiva foi presenciada por um parente.

Ao exame físico, apresentava-se com deformidade em rotação externa bilateralmente, associada a limitação funcional e dor. As imagens radiográficas evidenciaram fratura subcapital desviada bilateral, com sinais reabsortivos em ambos os colos femorais (fig. 1).

Após discussão clínica, optou-se pela artroplastia total do quadril bilateral pela via anterior direta. 0 procedimento foi feito em único tempo cirúrgico, pelo mesmo cirurgião, sem necessidade de troca de decúbito. Não foi necessário qualquer dispositivo de tração, foi feito em mesa cirúrgica tradicional em decúbito dorsal (fig. 2). Ambos os membros foram preparados em tempo único (fig. 3). Optou-se pela artroplastia total quadril bilateral não cimentada, os componentes usados foram a haste femoral Summit Tapered Hip System e componente acetabular Pinnacle Hip Solutions - DePuy Synthes. O par tribológico usado foi a cabeça de metal de diâmetro $32 \mathrm{~mm}$, associada a inserto de polietileno cross-linked do modelo ALTRX Altra-LinkedTM Polyethylene (fig. 4).

\section{Discussão}

Pacientes com epilepsia apresentam risco aumentado de fraturas de duas a seis vezes maior do que a população em geral, seja por quedas ou convulsões. ${ }^{4} \mathrm{O}$ uso de drogas antiepilépticas está associado a diminuição da densidade mineral óssea e risco

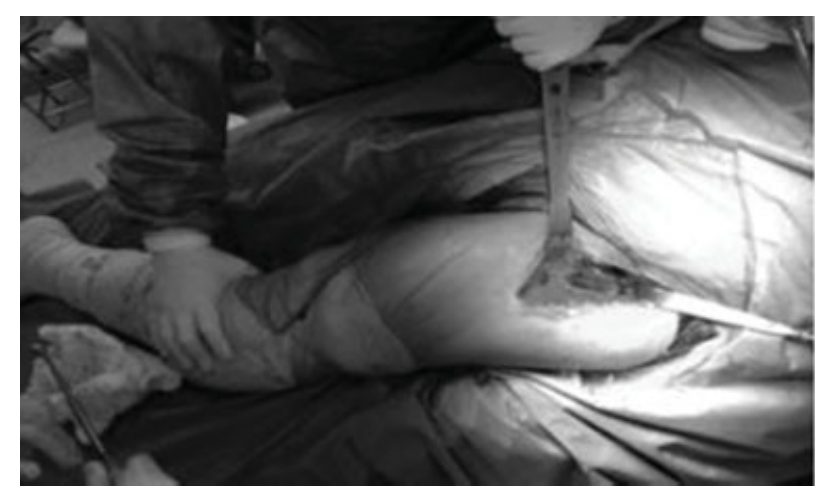

Fig. 2 Exposição femoral. 


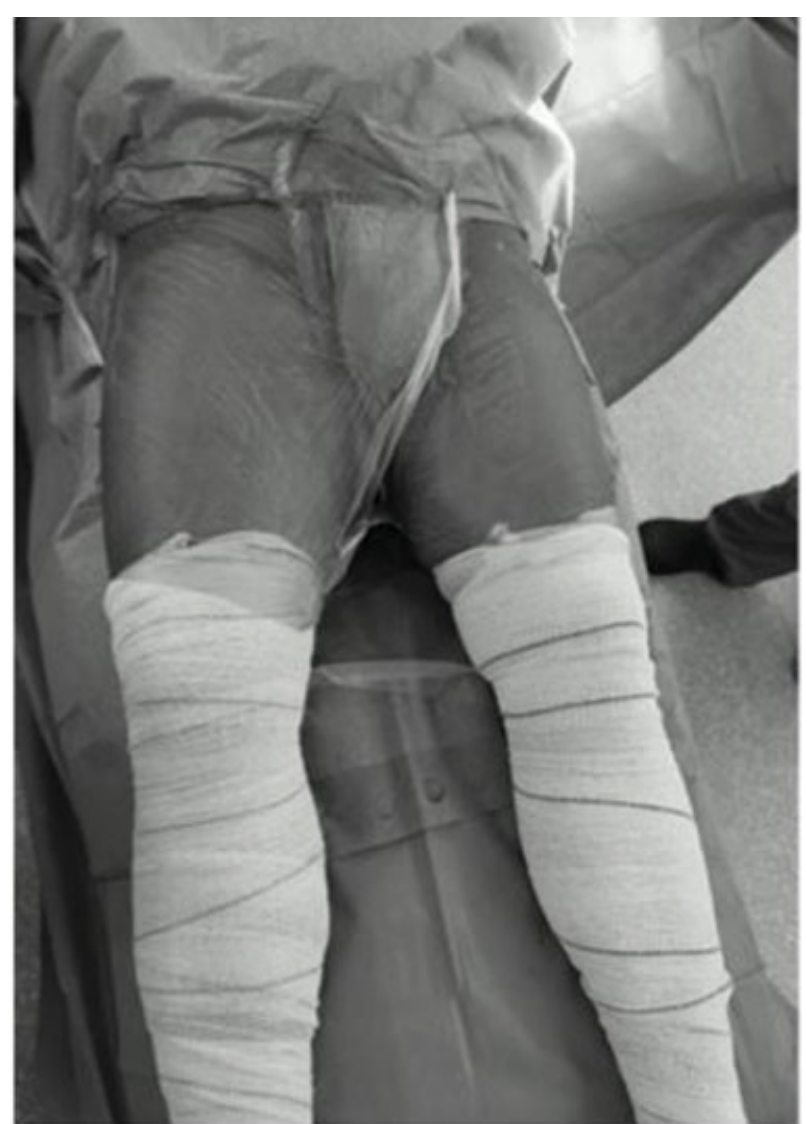

Fig. 3 Posicionamento e preparo do paciente.

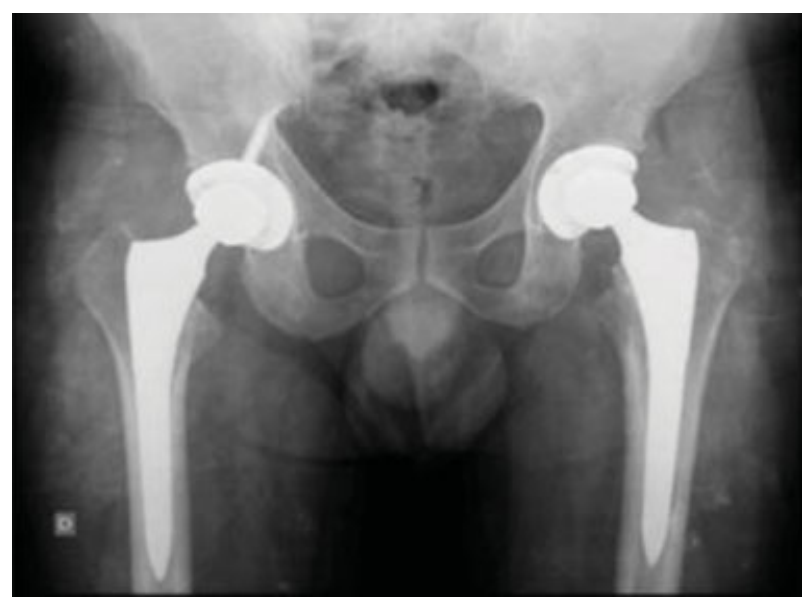

Fig. 4 Radiografia anteroposterior da bacia pós-operatória.

aumentado de fraturas. ${ }^{4,5}$ Homens de meia-idade com epilepsia mal controlada são os pacientes mais sujeitos às fraturas pós-convulsão, devido à musculatura mais desenvolvida. ${ }^{5}$ No caso apresentado, acredita-se que a causa da fratura tenha sido multifatorial, tanto pela osteopatia secundária à fenitoína como pelas contraturas tônico-clônicas, não foi a convulsão a causa, mas sim o evento desencadeante das fraturas.

A fixação da fratura do colo do fêmur é a opção de preferência em pacientes abaixo dos 60 anos; as artroplastias são indicadas em pacientes mais idosos ou em situações específicas. ${ }^{6}$ Sabe-se que a má redução da fratura do colo femoral pós-osteossíntese é fator preditivo mais importante para falha da fixação. Entretanto, não há indícios de que haja uma correlação direta entre o tempo de fixação versus a ocorrência de osteonecrose da cabeça femoral. ${ }^{6}$ Duckworth et al. ${ }^{7}$ descreveram correlação direta entre comorbidades que afetem a qualidade óssea e a falha da fixação nas fraturas desviadas do colo do fêmur em adultos jovens. Segundo os mesmos autores, pacientes acima de 40 anos com alterações da qualidade óssea são possíveis candidatos à artroplastia total do quadril devido ao elevado risco de falha da fixação.

No caso abordado, apesar de a literatura não evidenciar uma correlação direta entre o tempo de evolução e a ocorrência de osteonecrose da cabeça femoral, o tempo de evolução excessivamente prolongado associado a uma possível alteração osteometabólica e o alto risco de falha da fixação nos conduziram à artroplastia, apesar de temores quanto à possibilidade de luxação.

A artroplastia total de quadril bilateral pelo acesso anterior direito no mesmo tempo cirúrgico foi feita devido a única anestesia, menores custos financeiros, e conveniência quanto ao posicionamento. Além disso, Shao et al. ${ }^{8}$ relataram que a artroplastia total do quadril em tempo único apresenta menor risco cumulativo de complicações do que a artroplastia total do quadril em dois estágios, exceto a infecção periprotética.

A via de acesso é fundamental para a artroplastia do quadril, e devem-se levar em consideração diversos fatores, tais como a anatomia do paciente, a experiência do cirurgião, e a disponibilidade de material. 0 acesso anterior direto proporciona melhor reabilitação pós-operatória precoce, menor tempo de internação hospitalar, menor necessidade de opiáceos, e menor incisão cirúrgica. ${ }^{9,10}$ Entretanto, este acesso está associado com maior perda sanguínea, aumento do tempo cirúrgico, e uma longa curva de aprendizado. ${ }^{9,10}$ Os índices de luxação pós-artroplastia total do quadril em pacientes com fraturas do colo são maiores do que em pacientes submetidos ao mesmo procedimento devido à coxartrose. Entretanto, a escolha do acesso não altera esses índices de forma significativa. ${ }^{10}$ Segundo Marratt et al., ${ }^{10} \mathrm{o}$ acesso anterior apresenta índices de luxação similares ao acesso posterolateral com reparo capsular posterior, não há diferença na incidência de luxação. Dessa forma, a maior facilidade de preparo e posicionamento do paciente, o menor tempo de internação, a reabilitação pós-operatória precoce, e o domínio da técnica pelo cirurgião são possíveis justificativas à adoção do acesso anterior direto.

\section{Conclusão}

A ocorrência de fraturas em pacientes com epilepsia é aumentada por diversos fatores. $\mathrm{O}$ uso de medicações anticonvulsivantes que levem às alterações na qualidade óssea associado a episódios convulsivos pode acarretar fraturas do colo fêmur bilateral. ${ }^{4,5} \mathrm{~A}$ via de acesso ao quadril para artroplastia deve levar em consideração a anatomia do paciente, a experiência do cirurgião, e a disponibilidade de material. $\mathrm{O}$ acesso anterior direto é uma opção viável, com resultados clínicos semelhantes ao acesso posterolateral. 


\section{Conflito de Interesses}

Os autores declaram não haver conflito de interesses.

\section{Referências}

1 Grimaldi M, Vouaillat H, Tonetti J, Merloz P. Simultaneous bilateral femoral neck fractures secondary to epileptic seizures: treatment by bilateral total hip arthroplasty. Orthop Traumatol Surg Res 2009;95(07):555-557

2 Ribacoba-Montero R, Salas-Puig J. Simultaneous bilateral fractures of the hip following a grand mal seizure. An unusual complication. Seizure 1997;6(05):403-404

3 Vanderhooft E, Swiontkowski M. Bilateral femoral neck fractures following a grand mal seizure. Ann Emerg Med 1994;24(06): 1188-1191

4 Mattson RH, Gidal BE. Fractures, epilepsy, and antiepileptic drugs. Epilepsy Behav 2004;5(Suppl 2):S36-S40

5 Patil MM, Sahoo J, Kamalanathan S, Pillai V. Phenytoin Induced Osteopathy -Too Common to be Neglected. J Clin Diagn Res 2015;9 (11):OD11-OD12
6 Araujo TPF, Guimaraes TM, Andrade-Silva FB, Kojima KE, Silva JdosS. Influence of time to surgery on the incidence of complications in femoral neck fracture treated with cannulated screws. Injury 2014;45(Suppl 5):S36-S39

7 Duckworth AD, Bennet SJ, Aderinto J, Keating JF. Fixation of intracapsular fractures of the femoral neck in young patients: risk factors for failure. J Bone Joint Surg $\mathrm{Br}$ 2011;93(06): 811-816

8 Shao H, Chen CL, Maltenfort MG, Restrepo C, Rothman RH, Chen AF. Bilateral total hip arthroplasty: 1-stage or 2-stage? A metaanalysis. J Arthroplasty 2017;32(02):689-695

9 Meermans G, Konan S, Das R, Volpin A, Haddad FS. The direct anterior approach in total hip arthroplasty: a systematic review of the literature. Bone Joint J 2017;99-B(06):732-740

10 Maratt JD, Gagnier JJ, Butler PD, Hallstrom BR, Urquhart AG, Roberts KC. No difference in dislocation seen in anterior vs posterior approach total hip Arthroplasty. J Arthroplasty 2016; 31(9, Suppl)127-130 